\title{
Observational constraints on the progenitor of core-collapse supernovae
}

\author{
Nancy Elias-Rosa \\ INAF - Osservatorio Astronomico di Padova \\ Vicolo dell'Osservatorio 5, Padova I-35122, Italy \\ email: nancy.elias@inaf .oapd.it
}

\begin{abstract}
It is well-known that some massive stars evolve to an end state which results in the collapse of the stellar core, as the hydrostatic pressure can no longer support gravity, leading to powerful explosions called supernovae (SNe). Even with over 6000 known SNe, we have only direct information about the progenitor star for a handful of explosions. Here, I summarise the observational constraints of the massive progenitor stars of several core-collapse supernovae.
\end{abstract}

Keywords. (stars:) supernovae: general

Supernovae (SNe) are among the most influential events in the Universe. Their observational heterogeneity is a consequence of the different configuration of the progenitor stars at the moment of explosion, their energetics, angular momentum, and environment. If we understand the relationship between different SN properties and the stellar evolution in the local Universe, then SNe also become our best probe of mass loss, circumstellar structure, stellar evolution, and star formation rates throughout cosmic time. However, a fully consistent picture of the progenitor evolution and explosion is still missing.

Indirect clues about the SN origin can be derived from the interaction of the material dismissed during the explosion with dense circumstellar medium lost by the progenitor during its turbulent life. Still, the killer case is made with the direct identification of the star prior to explosion. Until the early 90s, only nearby events such SNe 1987A ( $50 \mathrm{kpc})$ and 1993J $(\sim 3.6 \mathrm{Mpc})$ have allowed the direct progenitor identification in pre-explosion images. In more recent years, it has been possible to resolve individual massive stars for distances up to $\sim 30-40 \mathrm{Mpc}$, thanks to the high resolution images provided mostly by the Hubble Space Telescope, but also by 8-m ground-based telescopes mainly provided by adaptive optics. Nonetheless, a final approach is visiting the $\mathrm{SN}$ field once we are confident the SN has faded, in order to verify if any source in the field has vanished, or at least has significantly weakened (e.g. see Maund \& Smartt 2009, Van Dyk et al. 2013). More than $6000 \mathrm{SNe}$ have been discovered but for only a handful of explosions we do have direct information about the progenitor. Although these lines of research involve very diverse methods and techniques, they share a unified scientific goal understanding the nature of the progenitors of SNe and their environments.

Following these or similar steps, direct detections or upper mass limits have been established for progenitors of some types of SNe:

- Type II-P: Based on the statistics of around 20 SNe II-P, it appears that all of these progenitors exploded in the red supergiant phase from stars with initial mass range of 8$17 \mathrm{M}_{\odot}$, as we would theoretically expect. However there has been no detection of a higher mass stars in the range $20-40 \mathrm{M}_{\odot}$, which should be the most luminous and brightest stars in these galaxies. This has led to the intriguing possibility that higher mass stars undergo core-collapse, but form black-holes which prevents much of the stellar mass escaping the 
explosion (Heger et al. 2003). Theoretically, such quenched, low energy explosions have been proposed. Our lack of detection of high mass progenitors could be evidence for this missing population (Kochanek et al. 2008, Smartt et al. 2009).

- Type II-L: Only in a couple of cases, e.g. SNe 2009hd (Elias-Rosa et al. 2011) and $2009 \mathrm{kr}$ (Elias-Rosa et al. 2010, Fraser et al. 2010), the progenitor has been identified and related with more yellow stars than expected by theoretical models, with estimated initial mass of between $18-25 \mathrm{M}_{\odot}$. However, a recent work argues that the progenitor of SN 2009kr is most probably a small compact cluster (Maund et al. 2015).

- Type IIb: These SNe likely originate from binary stripped progenitors with masses in the range 12-18 $\mathrm{M}_{\odot}$. The precursor of the prototype SN 1993J, was a B-type supergiant with $\sim 15 \mathrm{M}_{\odot}$ (e.g. Maund \& Smartt 2009).

- Type Ib/c: It has been proposed that they originate from the explosions of massive Wolf-Rayet (WR) stars, which should easily be detectable as they are very luminous and young. Along with the upper limits in most cases, it is still not possible to discriminate unambiguously between single WR stars or interacting massive stars in binary systems and the progenitors of Ib/c. Only for the recent case of iPTF13bvn (Cao et al. 2013) a possible WR was detected at the position of the SN. However, further analysis proposes instead a possible binary progenitor system (Bersten et al. 2014, Eldridge et al. 2015).

- Type Ibn: The precursor of SN 2006jc, prototype of this new subclass of SNe, was observed as a carbon-oxigen WR star embedded within a helium circumstellar medium with a mass between 60 and $100 \mathrm{M}_{\odot}$ (Pastorello et al. 2007).

- Type IIn: This class of SNe is related with Luminous Blue Variable (LBV) stars thanks to the progenitor detections of the SNe IIn 2005gl (Gal-Yam \& Leonard 2009), 2010j1 (Smith et al. 2011), or the debated SN 2009ip (e.g. Smith et al. 2010, Foley et al. 2011). Even so, and given the heterogeneity of this class, it is possible the existence of multiple precursor channels. The link between LBVs and type IIn SNe has been also possible through the invaluable study of the pre-SN photometric variability of the precursor stars, allowing us to characterise the final stages of the progenitors (e.g. Pastorello et al. 2013).

Although we are increasingly delimiting the SN explosion scenarios and mechanisms, there are still many unknowns. Each case we study provides new clues.

\section{References}

Bersten, M. C. et al., 2014, AJ, 148, 68

Cao, Y. et al., 2013, Ap. Lett., 775, 7

Eldridge, J. J. et al., 2015, MNRAS, 446, 2689

Elias-Rosa, N. et al., 2010, Ap. Lett., 714, 254

Elias-Rosa, N. et al., 2011, ApJ, 742, 6

Foley, R. J. et al., 2011, ApJ, 732, 32

Fraser, M. et al., 2010, Ap. Lett., 714, 280

Gal-Yam, A. \& Leonard, D. C., 2009, Nature, 458, 865

Heger, A. et al., 2003, ApJ, 591, 288

Kochanek, C. S. et al., 2008, ApJ, 684, 1336

Maund, J. R. \& Smartt, S. J. 2009, Science, 324, 486

Maund, J. R. et al., 2015, MNRAS, 447, 3207

Pastorello, A. et al., 2007, Nature, 447, 829

Pastorello, A. et al., 2013, ApJ, 767, 1

Smartt, S. J. et al., 2009, MNRAS, 395, 1409

Smith, N. et al., 2010, AJ, 139, 1451

Smith, N. et al., 2011, ApJ, 732, 63

Van Dyk, S. D. et al., 2013, ApJ, 772, 32 